\title{
A Theorem on Higher Bruhat Orders
}

\author{
S. Felsner and H. Weil \\ Fachbereich Mathematik und Informatik, \\ Freie Universität Berlin, \\ Takustrasse 9, 14195 Berlin, Germany \\ \{felsner,weil\}@inf.fu-berlin.de
}

\begin{abstract}
We show that inclusion order and single-step inclusion coincide for higher Bruhat orders $B(n, 2)$, i.e., $B(n, 2)=B_{\subseteq}(n, 2)$.
\end{abstract}

\section{Preliminaries}

Higher Bruhat orders were introduced by Manin and Schechtman [5] as generalizations of the weak Bruhat order on the symmetric group $S_{n}$. Further investigators of the subject are Voevodskij and Kapranov [6], Ziegler [7], Edelman and Reiner [1], [2], and Felsner and Weil [3]. We review the definition.

The set $[n]=\{1, \ldots, n\}$ is equipped with the natural linear order. The set of $s$-element subsets of $[n]$ is $\left(\begin{array}{c}{[n]} \\ s\end{array}\right)$. For $X \in\left(\begin{array}{c}{[n]} \\ s\end{array}\right)$ with $s \geq i \geq 1$ we let $X^{\lfloor i\rfloor}$ denote the set $X$ minus the $i$ th-largest element of $X$ (e.g., $\{3,5,8,9\}^{\lfloor 2\rfloor}=\{3,8,9\}$ ). For a set $P \in\left(\begin{array}{c}{[n]} \\ s+1\end{array}\right)$ the set of its $s$-element subsets $\left\{P^{\lfloor 1\rfloor}, P^{\lfloor 2\rfloor}, \ldots, P^{\lfloor s+1\rfloor}\right\}$ is called an $s$-packet, which we also denote by $P$, where this can be done unambiguously. Let $\mathcal{S}$ be a system of finite sets. The single-step inclusion order on $\mathcal{S}$ is the transitive closure of the relation $\lessdot$ on $\mathcal{S}$ defined by $S \lessdot S^{\prime}$ iff $S \subset S^{\prime}$ and $\left|S^{\prime} \backslash S\right|=1$.

Definition 1. A subset $A \subseteq\left(\begin{array}{c}{[n]} \\ s\end{array}\right)$ is called consistent if its intersection with any $s$ packet $P$ is either a beginning or an ending segment with respect to the lexicographic ordering of $P$, or equivalently if for any such packet $P$ and $1 \leq i<j<k \leq s+1$ the intersection of $A$ with $\left\{P^{\lfloor i\rfloor}, P^{\lfloor j\rfloor}, P^{\lfloor k\rfloor}\right\}$ is neither $\left\{P^{\lfloor i\rfloor}, P^{\lfloor k\rfloor}\right\}$ nor $\left\{P^{\lfloor j\rfloor}\right\}$.

The higher Bruhat order $B(n, s-1)$ is the set of consistent subsets of $\left(\begin{array}{c}{[n]} \\ s\end{array}\right)$ ordered by single-step inclusion. The partial order on this set by ordinary inclusion will be denoted by $B_{\subseteq}(n, s-1)$. 
In what follows it is preferable to work with $B(n, s)$ rather than with $B(n, s-1)$. To avoid confusion on the readers part we change letters from $s$ to $r$ (of course $s=r+1$ ).

$B(n, r)$ is a graded poset with unique minimal and maximal elements $\emptyset$ and $\left(\begin{array}{c}{[n]} \\ r+1\end{array}\right)$, respectively. The rank of a consistent set $A$ is $|A|$.

Further structural properties of higher Bruhat orders have been studied, in particular by Ziegler [7, Section 4]. He characterizes the pairs $(n, r)$ such that $B(n, r)$ is a lattice. Ziegler also shows that $B(n, r)=B_{\subseteq}(n, r)$ for $r=1$ and for $n-r \leq 4$ while $B(8,3) \neq$ $B_{\subseteq}(8,3)$. He left open the question whether $B(n, 2)$ is ordered by inclusion for $n>6$. Our main result is the affirmative answer to this question.

In the remainder of this introductory section we give alternative definitions for higher Bruhat orders and relate consistent subsets of $\left(\begin{array}{c}{[n]} \\ 3\end{array}\right)$ to arrangements of pseudolines and the notation customary in studies of such arrangements. Readers with an appropriate background may skip this part and proceed to Section 2 .

Manin and Schechtman defined the higher Bruhat order $B(n, r)$ as equivalence classes of admissible permutations of $\left(\begin{array}{c}{[n]} \\ r\end{array}\right)$. A permutation $\pi$ of the elements of $\left(\begin{array}{c}{[n]} \\ r\end{array}\right)$ is admissible, if the elements of every $r$-packet $P$ occur in $\pi$ in lexicographic or in reverse-lexicographic order. In the second case packet $P$ is called an inversion of $\pi$. Two admissible permutations $\pi$ and $\pi^{\prime}$ are equivalent if there is a sequence of admissible permutations $\pi=\pi_{0}, \pi_{1}, \ldots, \pi_{t}=\pi^{\prime}$ such that for $k=1, \ldots, t-1$ permutations $\pi_{k-1}$ and $\pi_{k}$ only differ by an adjacent transposition of two elements $X, X^{\prime}$ which are not contained in a common packet, i.e., two elements $X, X^{\prime}$ with $\left|X \cap X^{\prime}\right|<r-1$.

Ziegler [7] shows that admissible permutations of $\left(\begin{array}{c}{[n]} \\ r\end{array}\right)$ are equivalent iff they have the same sets of inversions and that a subset of $\left(\begin{array}{c}{[n]} \\ r+1\end{array}\right)$ is consistent iff it is the set of inversions of an admissible permutation of $\left(\begin{array}{c}{[n]} \\ r\end{array}\right)$. Hence, the two definitions for higher Bruhat orders are equivalent.

An admissible permutation of $\left(\begin{array}{c}{[n]} \\ 1\end{array}\right)$ is just a permutation of $[n]$ and consistency corresponds to transitivity of the inversion and the noninversion relation. Hence, the higher Bruhat order $B(n, 1)$ is the weak Bruhat order of permutations.

Admissible permutations of $\left(\begin{array}{c}{[n]} \\ 2\end{array}\right)$ have shown up in different facings. In studies on Coxeter groups they are the reduced decompositions of the reverse permutation. They are also the sequences of moves of a half-period (beginning with the identity) of a simple allowable sequence as defined by Goodman and Pollack [4]. Allowable sequences were introduced as a combinatorial model for arrangements of pseudolines. Of interest to us is the following statement: Admissible permutations of $\left(\begin{array}{c}{[n]} \\ 2\end{array}\right)$ encode simple marked arrangements of pseudolines and consistent subsets of $\left(\begin{array}{c}{[n]} \\ 3\end{array}\right)$ are in bijection with these arrangements.

Informally, a simple marked arrangements of pseudolines consists of $n$ curves which begin at the left side of the page and move across to the right side such that each pair of curves will cross and no three curves cross at a single point. Given an arrangement $\mathcal{A}$ label the pseudolines (= curves) such that at the left side they enter the picture in the natural order from bottom to top. Consider a triple $\left\{l_{i}, l_{j}, l_{k}\right\}$ of pseudolines with $i<j<k$ in $\mathcal{A}$. This triple can induce two combinatorially different arrangements. Either the crossing of $l_{i}$ and $l_{k}$ is above $l_{j}$ or below. In the first case the pairs $\{i, j\},\{i, k\}$, and $\{j, k\}$ appear in lexicographic order in the corresponding admissible permutation, in the second case the triple $\{i, j, k\}$ is an inversion. If the region enclosed by $l_{i}, l_{j}$, and 

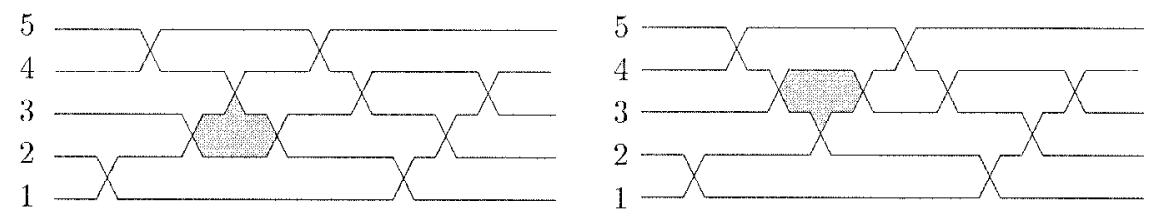

Fig. 1. Elementary flip at the shaded triangle.

$l_{k}$ is a triangle, we can apply an elementary flip to obtain another arrangement which combinatorially differs from the original one only in the orientation of this one triangle. This corresponds to a single step in the higher Bruhat order, i.e., to two consistent sets that differ in just one triple. Figure 1 shows the wiring diagrams corresponding to the consistent sets $\{145,234,235,245,345\}$ on the left and $\{135,145,234,235,245,345\}$ on the right.

Geometric interpretations of consistent sets have been the driving force for defining and investigating higher Bruhat orders (see [5]-[7] and [3]). Ziegler shows a bijection between consistent sets $A \in B(n, r)$ and uniform one-element extensions of the alternating oriented matroid $C^{n, n-r}$. By oriented matroid duality this also gives a bijection between consistent sets and one-element liftings of $C^{n, r}$.

Let $A \subseteq\left(\begin{array}{c}{[n]} \\ r+1\end{array}\right)$ be a consistent set and $X \in\left(\begin{array}{c}{[n]} \\ r-1\end{array}\right)$. Define an orientation $\rightarrow_{X}$ of the complete graph with vertex set $[n] \backslash A$. For two vertices $i, j$ with $i<j$ let

$$
j \rightarrow_{X} i \quad \Longleftrightarrow \quad X \cup\{i, j\} \in A \text {. }
$$

Claim T. The orientation $\rightarrow_{X}$ is transitive, i.e., the graph is a transitive tournament.

Proof. We argue by contradiction. Assume that the graph contains a cycle and hence a cycle on three vertices $i, j, k$, we assume $i<j<k$. We distinguish two cases:

(1) $i \rightarrow_{X} j \rightarrow_{X} k \rightarrow_{X} i$.

(2) $k \rightarrow_{X} j \rightarrow_{X} i \rightarrow_{X} k$.

The first case yields $X \cup\{i, j\} \notin A, X \cup\{i, k\} \in A$, and $X \cup\{j, k\} \notin A$, and the second case yields $X \cup\{i, j\} \in A, X \cup\{i, k\} \notin A$, and $X \cup\{j, k\} \in A$. Since $i<j<k$ it follows that in lexicographic order $X \cup\{i, j\}<_{\operatorname{lex}} X \cup\{i, k\}<_{\operatorname{lex}} X \cup\{j, k\}$. In both cases we obtain a contradiction to the consistency of $A$ with respect to the packet $X \cup\{i, j, k\}$.

Transitive tournaments have a unique topological sorting, hence, we get a collection of linear orderings or permutations $\alpha_{X}$ of $[n] \backslash X$ for every $X \in\left(\begin{array}{c}{[n]} \\ r-1\end{array}\right)$ associated to $A \in B(n, r)$. We call them local sequences.

If $r=2$, i.e., when we have an arrangement of pseudolines corresponding to $A$, a local sequence is a permutation $\alpha_{\{i\}}$ of $[n] \backslash\{i\}$. This permutation reports the order in which line $l_{i}$ is crossed by the other lines. Basically these are the local sequences of (un-)ordered switches associated to an allowable sequence by Goodman and Pollack [4]. 


\section{The Main Result}

In this section we show that the single-step order and the inclusion order on consistent subsets of $\left(\begin{array}{c}{[n]} \\ 3\end{array}\right)$ coincide.

Theorem 1. $B(n, 2)=B_{\subseteq}(n, 2)$ for all $n$.

To prove the result we show that for any two consistent sets $A \subset B \subseteq\left(\begin{array}{c}{[n]} \\ 3\end{array}\right)$ there is a consistent set $A^{\prime}$ satisfying $A \lessdot A^{\prime}$ and $A^{\prime} \subseteq B$. Iterating this argument we find a single-step chain $A=A_{0}, A_{1}, \ldots, A_{t}=B$ connecting $A$ and $B$ in $B(n, 2)$.

Given consistent sets $A \subset B \subseteq\left(\begin{array}{c}{[n]} \\ 3\end{array}\right)$ we call a triple $T \in B \backslash A$ a difference triple. From $A \subset B$ it follows that for all nondifference triples $T^{\prime}$ either $T^{\prime} \in A \cap B$ or $T^{\prime} \notin A \cup B$ holds. Let $\mathcal{A}$ be an arrangement of pseudolines with inversion set $A$. We will show that in $\mathcal{A}$ there is a triangular face $F$ such that the three lines bounding $F$ correspond to a difference triple. Call such a triple elementary. Given the triangle $F$ we can apply an elementary flip to obtain an arrangement $\mathcal{A}^{\prime}$ such that its inversion set $A^{\prime}$ has the desired properties, i.e., $A \lessdot A^{\prime}$ and $A^{\prime} \subseteq B$.

For $i<j<k$ the basis of the triple $\{i, j, k\}$ is the piece of line $l_{j}$ between the intersections with lines $l_{i}$ and $l_{k}$. Clearly an elementary triple has a basis which is an edge of the cell complex induced by $\mathcal{A}$. Call the basis of a triple which is an edge in the cell complex of $\mathcal{A}$ an elementary basis.

Let $\alpha_{i}$ denote the local sequence of line $l_{i}$ in $\mathcal{A}$, i.e., the permutation of $[n] \backslash\{i\}$ recording the order in which line $l_{i}$ is crossed by other lines. For a triple $\left\{i_{1}, i_{2}, i_{3}\right\}$ and $i_{1}<i_{2}$ recall the following equivalence:

$$
\left\{i_{1}, i_{2}, i_{3}\right\} \in A \quad \Longleftrightarrow \quad\left\{i_{1}, i_{2}\right\} \text { is an inversion of } \alpha_{i_{3}} .
$$

Lemma 2. There is a difference triple $T$ with an elementary basis.

Proof. Among all difference triples $\{i, j, k\}$ with $i<j<k$ choose one of minimal width $k-i$. Let this triple be $T=\{i, j, k\}$. From $T \notin A$ and $(*)$ we see that on line $l_{j}$ the intersection with line $l_{i}$ comes before the intersection with line $l_{k}$.

Claim A. For every $x$ between $i$ and $k$ in the local sequence $\alpha_{j}$ either $x<i$ or $x>k$.

Proof. Suppose $x$ with $i<x<k$ is between $i$ and $k$ on $\alpha_{j}$ denoted $i \prec x \prec k$. Now consider the order of $i, x, k$ on the local sequence $\beta_{j}$ of $B$. From $\{i, j, k\} \in B$ and (*) we obtain $k \prec i$ on $\beta_{j}$.

If $x \prec i$, on $\beta_{j}$ we obtain from ( $*$ ) that $\{i, j, x\}$ is a difference triple. If $i<x<j$ the width of this triangle is $j-i$, otherwise, if $i<j<x<k$ the width is $x-i$. In both cases this contradicts our choice of $\{i, j, k\}$ as a difference triangle of minimal width.

If $x \nprec i$, then $k \prec x$ on $\beta_{j}$. In this case $\{x, j, k\}$ is a difference triangle of width either $k-x$ or $k-j$. Again, this contradicts our choice of $\{i, j, k\}$ as a difference triangle of minimal width. 
Claim B. There exists an elementary basis on the segment of $l_{j}$ between the crossings with $l_{i}$ and $l_{k}$.

Proof. If $i$ and $k$ are adjacent elements of $\alpha_{j}$ we are done. Otherwise, by Claim A we can partition the elements between $i$ and $k$ into elements $x$ with $x<i$ and elements $y$ with $y>k$. For an $x$ we note that from $i \prec x$ on $\alpha_{j}$ we obtain $\{x, i, j\} \in A$. Hence, $\{x, i, j\} \in B$, i.e., $i \prec x$ on $\beta_{j}$. Since $k \prec i$ on $\beta_{j}$ the triple $\{x, j, k\}$ is a difference triple. For an element $y$ we obtain by an analogous argument that $\{i, j, y\}$ is a difference triple.

If the element to the right of $i$ on $\alpha_{j}$ is a $y$, the difference triple $\{i, j, y\}$ has an elementary basis and we are done. If the element to the left of $k$ on $\alpha_{j}$ is an $x$, the difference triple $\{x, j, k\}$ has an elementary basis and we are again done. If both these conditions fail, then we find an adjacent pair $(x, y)$ with $x<i$ and $y>k$ on $\alpha_{j}$. On $\alpha_{j}$ we have $i \prec x \prec y \prec k$ while by the above considerations $y \prec k \prec i \prec x$ on $\beta_{j}$. This shows that $\{x, j, y\}$ is a difference triple with an elementary basis.

This completes the proof of the lemma.

We now consider a wiring diagram for $\mathcal{A}$. Wiring diagrams are closely related to allowable sequences. Informally, a wiring diagram is a drawing of $\mathcal{A}$ in which the edges are associated to horizontal wires (see Fig. 1). For an edge $e$ of $\mathcal{A}$ we say $e$ is on wire $w$ if the horizontal portion of $e$ is on wire $w$. Let $\{i, j, k\}$ be a difference triple with elementary basis such that the basis of $\{i, j, k\}$ is on the highest wire that contains elementary bases in the diagram.

Lemma 3. The triple $\{i, j, k\}$ defined in the preceding paragraph is an elementary triple.

Proof. Since the basis of $\{i, j, k\}$ is elementary any line $l_{x}$ crossing the triangle of the three lines $l_{i}, l_{j}, l_{k}$ enters the triangle through line $l_{i}$ and leaves the triangle through line $l_{k}$. It follows that $i<x<k$.

If $i<x<j$, then $\{i, x, j\} \in A$, hence, $\{i, x, j\} \in B$ and on $\beta_{i}$ we have $j \prec x$. With $k \prec j$ on $\beta_{i}$ this shows that $\{i, x, k\}$ is a difference triple. Similarly, if $j<x<k$, then $\{j, x, k\} \in A$ and $\{j, x, k\} \in B$. Considering $\beta_{k}$ we see that again $\{i, x, k\}$ is a difference triple.

Let $F$ be the face of $\mathcal{A}$ above the edge on $l_{j}$ corresponding to the basis of $\{i, j, k\}$. The boundary of $F$ consists of the basis $b$ and edges $e_{0}, \ldots, e_{t}$ in clockwise order. Figure 2 shows a generic sketch of the situation. Note that in the wiring diagram of $\mathcal{A}$ the edges $e_{0}, \ldots, e_{t}$ are all on the wire above the wire of $b$.

Claim C. If $t>1$, then one of the edges $e_{1}, \ldots, e_{t-1}$ is an elementary basis.

Claim $C$ gives a contradiction to the choice of the triple $\{i, j, k\}$ when $t>1$. Therefore, $t=1$ and face $F$ is a triangle in $\mathcal{A}$ This shows that $\{i, j, k\}$ is an elementary triple. To prove the lemma it thus suffices to prove the claim. 


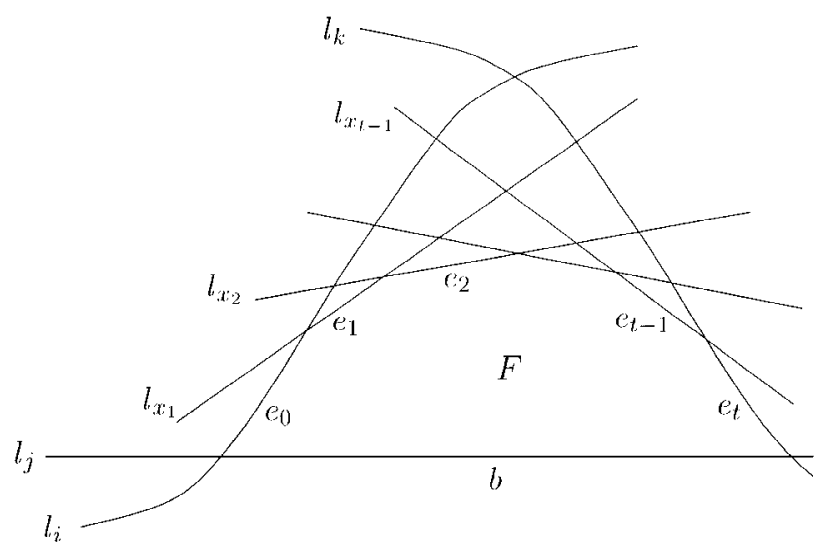

Fig. 2. Face $F$ above the elementary basis $b$.

Proof. If $t=2$ let $l_{x}$ be the supporting line of $e_{1}$. From the above considerations we know that $\{i, x, k\}$ is a difference triple. The basis of the triple is edge $e_{1}$, hence is elementary.

If $t>2$ let $l_{x_{s}}$ be the supporting line of edge $e_{s}$ for $s=1, \ldots, t-1$. Note that $i \prec x_{s+1} \prec k$ on $\alpha_{x_{s}}$ and $k \prec i$ on $\beta_{x_{s}}$. Therefore, at least one of $\left\{i, x_{s}, x_{s+1}\right\}$ and $\left\{x_{s}, x_{s+1}, k\right\}$ is a difference triple. For $s=1, \ldots, t-2$ let $p_{s}$ be the vertex of $e_{s} \cap e_{s+1}$. Color $p_{s}$ red if $\left\{i, x_{s}, x_{s+1}\right\}$ is a difference triple and blue otherwise.

If $p_{1}$ is a red vertex, then $e_{1}$ is an elementary basis. If $p_{t-2}$ is a blue vertex, then $e_{t-1}$ is an elementary basis. Now assume that $p_{1}$ is blue and $p_{t-2}$ is red, then there is some $s$ such that $p_{s}$ is blue and $p_{s+1}$ is red. Note that $x_{s}<x_{s+1}<x_{s+2}$ and $x_{s} \prec x_{s+2}$ on $\alpha_{x_{s+1}}$. From the definitions of red and blue vertices we obtain $i \prec x_{s}$ and $x_{s+2} \prec i$ on $\beta_{x_{s+1}}$. Hence, $\left\{x_{s}, x_{s+1}, x_{s+2}\right\}$ is a difference triple with elementary basis $e_{s+1}$. This proves the claim and completes the proof of the lemma.

Lemmas 2 and 3 prove the theorem.

\section{Reorientations}

If we fix a consistent set $A_{1}$ in $B(n, r)$ we can reorient this order to obtain $B^{A_{1}}(n, r)$. For $A_{2} \in B(n, r)$ we define the corresponding reoriented inversion set $A_{2}{ }^{A_{1}}$ in $B^{A_{1}}(n, r)$ as the symmetric difference $A_{2} \triangle A_{1}$. The order relation of $B^{A_{1}}(n, r)$ is the single-step order on these reoriented inversion sets. Again we define $B_{\subseteq}^{A_{1}}(n, r)$ as the order on the same elements with inclusion as order relation. Ziegler [7] initiated the study of reoriented higher Bruhat orders. He showed that reorientations lack some of the structure of higher Bruhat orders. In particular he shows that while $B(6,3)$ is ordered by inclusion there is a consistent set $A \in B(6,3)$ such that $B^{A}(6,3)$ is not ordered by inclusion. He shows that $B^{A}(6,3)$ is not even bounded.

The following example shows a similar "bad" behavior for reorientations of $B(6,2)$. Let $\mathcal{A}_{1}$ and $\mathcal{A}_{2}$ be the simple arrangements shown in Fig. 3. Both arrangements have 

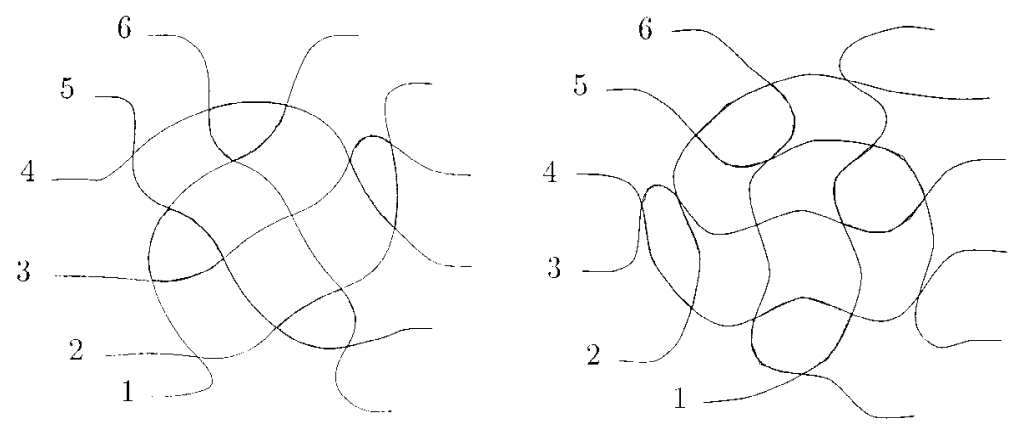

Fig. 3. Two arrangements $\mathcal{A}_{1}$ and $\mathcal{A}_{2}$ with corresponding triangular faces.

exactly four triangular faces determined by the following sets of lines $\{1,3,5\},\{1,4,6\}$, $\{2,3,4\}$, and $\{2,5,6\}$, moreover, the orientation of these triangles is the same in both arrangements. It follows that starting from $\mathcal{A}_{1}$ every possible triangular flip leads to an arrangement with more 3-element sets of lines being oriented differently from their orientation in $\mathcal{A}_{2}$. Hence, if we orient $B(6,2)$ away from the consistent set $A_{1}$ corresponding to $\mathcal{A}_{1}$ there is no single element step toward the consistent set $A_{2}$ corresponding to $\mathcal{A}_{2}$. Hence, every chain (in the inclusion order) from $A_{1}$ to the complement $\overline{A_{1}}$ through $A_{2}$ has length less than $\left(\begin{array}{l}6 \\ 3\end{array}\right)$. This example shows:

(1) Single step inclusion and inclusion are not identical for the reorientation $B^{A_{1}}(6,2)$ of $B(6,2)$ and hence $B(n, 2)$ for all $n \geq 6$.

(2) Both, $A_{1}$ and $A_{2}$ admit no single step going down in $B^{A_{1}}(6,2)$, hence the reorientation is unbounded.

(3) An arrangement of pseudolines is not (necessarily) determined by the orientations of its triangular faces. Since the arrangements $\mathcal{A}_{1}$ and $\mathcal{A}_{2}$ are realizable the same holds for arrangements of lines.

\section{References}

1. P. Edelman and V. Reiner, Free arrangements and rhombic tilings, Discrete Comput. Geom., 15 (1996), 307-340.

2. P. Edelman and V. Reiner, Erratum to free arrangements and rhombic tilings, Discrete Comput. Geom., 17 (1997), 359.

3. S. Felsner and H. Weil, Sweeps, arrangements and signotopes, Technical Report, FU-Berlin, 1998.

4. J. E. Goodman and R. Pollack, Semispaces of configurations, cell complexes of arrangements, J. Combin. Theory Ser. A, 37 (1984), 257-293.

5. Y. Manin and V. Schechtman, Arrangements of hyperplanes, higher braid groups and higher Bruhat orders, in Algebraic Number Theory - in Honour of K. Iwasawa, J. C. et al., eds., Advanced Studies in Pure Mathematics, vol. 17, Kinokuniya Company/Academic Press, New York, 1989, pp. 289-308.

6. V. Voevodskij and M. Kapranov, Free $n$-category generated by a cube, oriented matroids, and higher Bruhat orders, Functional Anal. Appl., 2 (1991), 50-52.

7. G. Ziegler, Higher Bruhat orders and cyclic hyperplane arrangements, Topology, 32 (1993), 259-279.

Received April 7, 1998. 